\title{
Quasi-Classical Gravity Effect on Neutrino Oscillations in a Gravitational Field of a Heavy Astrophysical Object
}

\author{
Jonathan Miller ${ }^{1}$ and Roman Pasechnik ${ }^{2}$ \\ ${ }^{1}$ Departamento de Física, Universidad Técnica Federico Santa María, Casilla 110-V, 2930123 Valparaíso, Chile \\ ${ }^{2}$ Theoretical High Energy Physics, Department of Astronomy and Theoretical Physics, Lund University, \\ Sölvegatan 14A, 223-62 Lund, Sweden
}

Correspondence should be addressed to Jonathan Miller; jonathan.miller@usm.cl

Received 1 December 2014; Revised 4 March 2015; Accepted 1 May 2015

Academic Editor: Srubabati Goswami

Copyright (C) 2015 J. Miller and R. Pasechnik. This is an open access article distributed under the Creative Commons Attribution License, which permits unrestricted use, distribution, and reproduction in any medium, provided the original work is properly cited. The publication of this article was funded by SCOAP S $^{3}$

In the framework of quantum field theory, a graviton interacts locally with a quantum state having definite mass, that is, the gravitational mass eigenstate, while a weak boson interacts with a state having definite flavor, that is, the flavor eigenstate. An interaction of a neutrino with an energetic graviton may trigger the collapse of the neutrino to a definite mass eigenstate with probability expressed in terms of PMNS mixing matrix elements. Thus, gravitons would induce quantum decoherence of a coherent neutrino flavor state similarly to how weak bosons induce quantum decoherence of a neutrino in a definite mass state. We demonstrate that such an essentially quantum gravity effect may have strong consequences for neutrino oscillation phenomena in astrophysics due to relatively large scattering cross sections of relativistic neutrinos undergoing large angle radiation of energetic gravitons in gravitational field of a classical massive source (i.e., the quasi-classical case of gravitational Bethe-Heitler scattering). This graviton-induced decoherence is compared to decoherence due to propagation in the presence of the Earth matter effect. Based on this study, we propose a new technique for the indirect detection of energetic gravitons by measuring the flavor composition of astrophysical neutrinos.

\section{Introduction}

A theoretical extrapolation of the fundamental quantum mechanics concepts to Einstein's gravity suffers from major difficulties with quantization of space-time, ultraviolet behavior and nonrenormalizability of the resulting theory (for more details, see $[1,2]$ and references therein). A wealth of theoretical studies have been presented in the literature and many different quantum gravity models have been developed. However, no conclusive statement about the true quantum nature of gravity has been made. Only a real experiment can settle the longstanding confusion between the different approaches and provide guidance in developing the correct underlying theory.

Typically, in the standard quantum field theory framework which unifies three of four basic forces of nature, the quantum gravity effects are disregarded as being phenomenologically irrelevant at energy scales much smaller than the Planck scale, $M_{\mathrm{Pl}} \sim 10^{19} \mathrm{GeV}$. Moreover, due to enormous suppression, quantum gravity effects are often referred to as nearly unobservable [3-5]. While observing a single graviton directly may be impossible, it is not impossible to find an indirect evidence for quantum gravity. For an overview of potential phenomenological opportunities for indirect signatures of quantum gravity, see [6-9]. Nevertheless, our understanding of the quantum nature of gravity suffers from the lack of accessible sources of information.

In this paper, we propose a new approach for indirect experimental studies of (local) quantum gravity interactions based upon an effect of the large angle energetic gravitational Bremsstrahlung (or Gravi-strahlung, in short) off an astrophysical neutrino passing through an external classical gravitational potential on neutrino oscillation observables. This process, known as the gravitational Bethe-Heitler (GBH) process, can be considered in the quasi-classical approximation for large angle and/or large energy graviton emission; 
that is, the Born approximation is sufficient. Such a process may happen with a rather high probability, such as in the case of an astrophysical neutrino scattering off a massive source of classical gravitational field (like a star, black hole, dark matter distribution, or galaxy). In quantum mechanics, the latter process may serve as a direct quantum measurement of the microscopic properties of the gravitational field at astrophysical scales.

Quasi-Classical Gravity. In the limit of weak gravity, the quasi-classical approximation to quantum gravity is a valid framework. In this case, the graviton field is a correction determined on the flat Minkowskian background and the metric operator in the Heisenberg representation is given by $\widehat{g}_{\mu \nu}=\eta_{\mu \nu}+\widehat{h}_{\mu \nu}$. Here, the $c$-number part $\eta_{\mu \nu}$ is the Minkowski metric and $\widehat{h}_{\mu \nu}$ is the graviton arising after the quantization procedure. The Einstein-Hilbert action provides the mechanism for virtual gravitons to propagate in the flat space-time and to interact with one another in the quantum case as an analog of the standard QED picture of the Coulomb field around an electric charge. These virtual gravitons should be distinguished from real gravitons which are radiated off an accelerated massive body and their coherent wave packets correspond to gravitational waves in the classical limit. A "cloud" of virtual gravitons around a static massive body can be reinterpreted geometrically in terms of a deviation from the flat metric (or curvature) in Einstein's classical relativity $[10,11]$ (the background must be chosen to be flat since only in this case is it possible to use the Casimir operators of the Poincaré group and show that the quanta have spin two and rest mass zero, thus being identified as gravitons).

A graviton couples to the full energy-momentum tensor. From the quantum mechanical point of view, we work in the mass eigenstate basis where the Hamiltonian of local quantum gravitational interactions has a diagonal form and identify the particle mass eigenstates with gravitational eigenstates (due to equivalence of gravitational and inertial mass). In this approach, higher Fock states are created by the graviton creation operator acting on a particle mass eigenstate. By measuring the quasi-classical graviton cross section and deviations from it, we would be engaging in the first investigations of the deeper quantum gravity theory similar to how electroweak $\nu$ - $A$ measurements provided the first investigation of the deeper quantum Weinberg-Salaam theory.

\section{Decoherence of Neutrino State}

Generically, weakly interacting neutrinos can be considered as an efficient carrier of information across the universe as they are not absorbed or scattered by interstellar mediums. In practice, this unique property of neutrinos enables us to utilize them for large-scale astrophysical "experiments," such as searching for possible tiny signatures of Lorentz invariance violation [12], testing general relativity [13] and quantum mechanics [14-16], testing the equivalence principle $[17,18]$, and testing minimal length models $[19,20]$. Ultimately, it is possible to identify an extraterrestrial large-scale quantum experiment where neutrinos "change" their quantum state due to a local quantum gravity process (in terms of local graviton coupling to a fundamental matter particle) and further convey information about such a process unchanged through the cosmological medium to the Earth.

2.1. Propagation Decoherence. The traditional source of decoherence typically referred to in astrophysical neutrino oscillations studies can be called propagation decoherence. This is when the distance that a neutrino travels exceeds the neutrino oscillation length. In this case, the neutrino mass states have separated so that they no longer interfere at large distances from the production point. This source of decoherence depends on the energy resolution of the detection process, the energy of the neutrino, the masses of the neutrino mass states, and other details of the production and detection processes. In neutrino experiments, the time between neutrino production and detection is normally not measured. In a real experiment, this means that beyond the neutrino oscillation length the propagating neutrino mass states no longer interfere during the interaction process in a detector [21, 22]. For cosmic/astrophysical neutrinos, in some cases and for some processes, this decoherence effect is irrelevant $[23,24]$.

2.2. Classical Diósi-Penrose Decoherence. The role of classical Einstein's gravity in quantum mechanics is under extensive consideration in the literature and may be sizeable under certain conditions. As was claimed in [25], the gravityinduced quantum state reduction can be tested by observing the neutrino flavor oscillations at cosmological distances, while in [26] it was regarded as practically undetectable. This classical gravity effect on real-time evolution of a quantum state composed of several mass eigenstates was initially considered by Diósi [27-29] and Penrose [30]. In the classical gravity limit, the latter can be approximated by a change in the phase of the flavor wave function which appears mainly due to a nondegeneracy of neutrino mass eigenstates; that is, $\Delta m_{i j}^{2} \equiv m_{j}^{2}-m_{i}^{2} \neq 0$, where $m_{j}$ is the mass of the mass eigenstate $j$. This is caused by different mass states traveling along different geodesics in curved space-time and the whole effect gradually accumulates over large cosmological distances [31]. This is the essence of classical decoherence of a neutrino flavor state which is typically regarded as a probe for neutrino wave function collapse models and, more generally, alternatives to conventional (linear) quantum mechanics [32]. Instead, we consider another possible decoherence mechanism of a neutrino flavor state triggered at the quantum level by a single local graviton-neutrino interaction. Let us discuss this phenomenon in detail.

2.3. Quantum Decoherence. We expect elementary particles in the mass basis to be gravitational eigenstates of the Hamiltonian of quantum gravitational interactions in the same way as leptons and quarks are weak eigenstates in the flavor and CKM basis, respectively. The advantage of the neutrinos which we exploit here is that they interact via the weak force and that neutrino mass and flavor eigenstates are not the same and that they propagate at cosmological 
distances/times. For particles whose flavor and mass eigenstates are identical this technique would not work to identify that a graviton induced quantum mechanical interaction had happened, which means that the neutrino is a unique carrier of astrophysical quantum gravity interactions.

Consider first a relativistic neutrino state propagating in the gravitational potential of a supermassive black hole, dark matter halo, or another massive system. These not only are sources of strong gravitational fields but could also be significant sources of astrophysical neutrinos. Suppose now that at the quantum level a graviton interacts only with a definite mass state (or gravitational mass eigenstate) $a=$ 1,2 or 3 . This is equivalent to saying that definite mass eigenstates (the propagating states) are conserved by the quantum gravity Hamiltonian while superpositions, such as the flavor eigenstates, are not [33]. Note, the astrophysical neutrinos are initially produced in electroweak processes (e.g., in $\mathrm{SNe}$ processes) in a definitive flavor state, $f=e, \mu$ or $\tau$, which are coherent superpositions of mass eigenstates. In an astrophysical environment, a high-energy graviton can interact only with a definite mass component of the neutrino wave function, thus causing quantum decoherence of the neutrino which is in a superposition of mass states, effectively "converting" it into a definitive mass eigenstate. This neutrino is quantum mechanically observed as being in a definite mass state. This means that between the production in an AGN or supernova $(\mathrm{SNe})$ or other astrophysics source and the detection in an Earth based detector, the neutrino which was observed by the graviton exists in a definite mass state. This is independent from propagation decoherence.

The neutrino is "converted" to mass state with a probability $P_{v_{f} \rightarrow v_{a}}=\left|\Psi_{v_{f} \rightarrow v_{a}}\right|^{2}$, given in terms of the corresponding wave function $\Psi_{v_{f} \rightarrow v_{a}}$ which projects out a flavor state $v_{f}$ onto a mass state $v_{a}$ and is typically expressed in terms of the corresponding PMNS mixing matrix element, $\Psi_{v_{f} \rightarrow v_{a}} \equiv V_{a f}$. The considered effect is different from other known classical decoherence sources emerging due to a mere propagation (without a hard graviton radiation) in classical gravitational potential and/or neutrino propagation in flat space-time. The effect under consideration is a straightforward consequence of fundamental time-energy uncertainty relation for the real hard Gravi-strahlung and should be taken into account in studies of astrophysical neutrino oscillations.

The amplitudes of typical quasi-classical gravity scattering processes which may lead to the quantum decoherence effect under certain conditions can be represented as follows:

$$
\begin{aligned}
& A_{v_{f} \rightarrow v_{a}}^{(G), 1}=\Psi_{v_{f} \rightarrow v_{a}} A^{(G)}\left(v_{a}+G \longrightarrow v_{a}+G\right), \\
& A_{v_{f} \rightarrow v_{a}}^{(G), 2}=\Psi_{v_{f} \rightarrow v_{a}} A^{(G)}\left(v_{a}+M \longrightarrow v_{a}+G+M\right) .
\end{aligned}
$$

Here, $M$ is a source of strong classical gravitational fields, such as a massive star or a black hole. The first amplitude corresponds to the gravitational Compton scattering of a neutrino mass state off a real graviton in the medium, the second amplitude represents the GBH scattering of a neutrino in gravitational mass state off a classical heavy source $M$ (with energetic graviton radiation). Clearly, a mass eigenstate $v_{a}$ "produced" in this interaction due to decoherence does not undergo oscillation until it interacts weakly with normal matter (e.g., in an Earth detector) by means of $W, Z$-exchange. Therefore, quantum decoherence may have an nonnegligible effect on neutrino oscillation observables, along with other existing sources of classical decoherence and medium matter effects $[34,35]$. Explicitly, oscillation characteristics of neutrinos coming from, for example, a vicinity of the Galactic Center, may differ from vacuum oscillations. The latter case could be where a source of neutrinos is "nearby" but where there is no massive objects between the source and the Earth (nor significant variations in dark matter density). Such neutrinos, if identified, could be used as a control sample.

In a sense, the quantum gravity-induced decoherence of a definite flavor state described above is in close analogy to the weak-induced decoherence of a definite mass state. For example, $W, Z$ bosons interact only with a coherent flavor state inducing a "conversion" of a definite mass state into a definite flavor state. Namely, a neutrino in a mass eigenstate $v_{a}$ turns into a flavor eigenstate $v_{f}$ through an interaction with the virtual $Z, W$-bosons propagating in the $t$-channel, that is, four different reactions, are possible as follows:

$$
\begin{aligned}
& A_{v_{a} \rightarrow v_{f}}^{(w), 1}=\Psi_{v_{a} \rightarrow v_{f}} A^{(w)}\left(v_{f}+l_{f}^{\prime} \longrightarrow v_{f}^{\prime}+l_{f}\right), \\
& A_{v_{a} \rightarrow v_{f}}^{(w), 2}=\Psi_{v_{a} \rightarrow v_{f}} A^{(w)}\left(v_{f}+l_{f}^{\prime} \longrightarrow v_{f}+l_{f}^{\prime}\right), \\
& A_{v_{a} \rightarrow v_{f}}^{(w), 3}=\Psi_{v_{a} \rightarrow v_{f}} A^{(w)}\left(v_{f}+N \longrightarrow v_{f}+X\right), \\
& A_{v_{a} \rightarrow v_{f}}^{(w), 4}=\Psi_{v_{a} \rightarrow v_{f}} A^{(w)}\left(v_{f}+N \longrightarrow l_{f}+X\right),
\end{aligned}
$$

such that $\Psi_{v_{a} \rightarrow v_{f}}=\Psi_{v_{f} \rightarrow v_{a}}^{*}$. Here, a definitive mass state which may exist due to previous hard neutrino-graviton interaction or due to the resonance MSW effect $[34,35]$ is "converted" back into a flavor state which may undergo oscillation. It is important to note that because the neutrino is not likely to interact weakly between the source and the Earth, if the neutrino is in a definitive mass state induced by the hard neutrino-graviton scattering event which occurred long before it arrives at the Earth it will still be in the definitive mass state at the Earth. The distance between a hard neutrino-graviton scattering event and detection event is not important.

In the case of vacuum neutrino oscillations, the traveling neutrino is not in a definitive mass eigenstate but is rather in a superposition of mass eigenstates which evolves when the neutrino travels in space-time. Then, with respect to the weak interactions, the nondiagonal $\Psi_{v_{f} \rightarrow v_{f^{\prime}}}$ transition amplitude between two flavor states $f$ and $f^{\prime}$ is given by [36]

$$
\Psi_{v_{f} \rightarrow v_{f^{\prime}}}=\sum_{j} V_{f^{\prime} j} e^{-i\left(m_{j}^{2} / 2 E_{\nu}\right) L} V_{f j^{\prime}}^{*}
$$

here $L$ is the distance from where the neutrino was created in a definite flavor eigenstate $v_{f}$, and $E_{v}$ is the energy of the neutrino. Analogically, for neutrino-graviton interactions 
the $\Psi_{v_{f} \rightarrow v_{a}}$ transition amplitude between a flavor state $f$ and a mass state $a$ can be written as

$$
\Psi_{v_{f} \rightarrow v_{a}}=e^{-i\left(m_{a}^{2} / 2 E_{\nu}\right) L} V_{a f}
$$

which means that the probability for a given flavor neutrino state $f$ to decohere by transforming into a mass state $a$ due to a hard graviton-neutrino interaction, given by $P_{v_{f} \rightarrow v_{a}}^{(G)} \sim$ $\left|A_{v_{f} \rightarrow v_{a}}^{(G)}\right|^{2}=\left|\Psi_{v_{f} \rightarrow v_{a}}\right|^{2}\left|A^{(G)}\right|^{2}$, is independent of the neutrino mass, $m_{a}$, the mass splitting, $\Delta m_{a b}$, and the distance from the neutrino source, $L$. The dependence on the relativistic neutrino energy, $E_{v} \gg m_{a}$, for a given scattering comes from the neutrino mass state scattering amplitude squared, $\left|A^{(G)}\right|^{2}$ (for more details, see the next section).

2.3.1. Differences from Other Sources of Decoherence. Contrary to the Penrose-Diósi effect of classical decoherence [27-30], the quantum decoherence of a neutrino flavor state happens at small space-time scales, $\Delta l_{\text {dec }}$, which are much smaller than the neutrino wave length scale: $\Delta l_{\text {dec }} \ll$ $L_{v}$, due to the quantum nature of the tree-level gravitonneutrino interaction. As an additional significant difference, the quantum decoherence effect is not sensitive to the mass differences of the mass eigenstates, or to $\Delta m_{i j}^{2}$, while they are crucial for and determine the classical decoherence of the neutrino flavor state at large separations, $\Delta l_{\mathrm{dec}} \gg L_{v}$. Most importantly, quantum decoherence provides us with a key for phenomenological verification of quantum gravity models with possible deviations from quasi-classical gravity through measurement of neutrino oscillation characteristics.

The proposed effect is also different from the standard propagation decoherence (see Figure 2). In propagation decoherence, the neutrino mass states are separated in time and/or space and so the local weak interaction (the detection process) observes an incoherent sum of the propagating mass states in a given space-time point. In quantum decoherence, the neutrino exists only within a given mass eigenstate after being "observed" by the hard graviton (e.g., in the quantum processes of hard GBH or Compton scattering; see below). This difference is important. Indeed, while a flux of neutrinos which have undergone quantum decoherence is observed by a weak interaction in an Earth-based detector as an incoherent sum of the mass states, they do not experience a change of potential induced by matter (e.g., the MSW effect) as an incoherent sum of mass states. Namely, the neutrino which has not undergone quantum decoherence experiences matter as a superposition of mass states, while the neutrino which has undergone quantum decoherence would not experience matter as a superposition of mass states. Also, it is possible that a neutrino passing through densities which change nonadiabatically might demonstrate interference phenomena as presented in [23]. As we will explicitly demonstrate below, such a difference between the quantum and propagational decoherence in the presence of the Earth matter effect may be observable and is important in studies of astrophysical neutrino oscillations.
2.4. Decoherence in the Presence of the Earth Matter Effect. We would like to note that while the flux from quantum (gravitational) decoherence is a flux of pure mass eigenstates as noted, the important difference is that in the propagation decoherence case the flux is not of pure mass eigenstates, but rather decoherent (spatially separated) mass eigenstates. No quantum measurement of the state of these neutrinos has taken place, and the neutrino still exists as a superposition of mass states (just no longer with off diagonal elements in the density matrix). While these two situations are exactly the same when detected in the case where the flux is detected without passing through matter, in the case where the flux passes through matter, the regeneration which the neutrino flux experiences is different for the two cases. In the quantum gravitational decoherence case, the neutrino flux experiences regeneration as fluxes of neutrinos in pure mass eigenstates. However, in the propagation decoherence case, the neutrino flux experiences regeneration as a superposition of mass eigenstates; individual actual neutrinos continue to exist in a spatially separated quantum superposition of mass eigenstates. These spatially separated quantum superpositions experience the potential of the Earth. Simulation was done to demonstrate the possible size of this effect due to the difference in regeneration in the two cases (details below). In the simulation the neutrino is considered to have experienced propagation decoherence and the exact distance the neutrino travelled is not important (as long as it fulfils the conditions in [24]); in this work we consider relatively small distances $(<1000 \mathrm{kpc})$ since we do not explicitly consider differentiating quantum decoherence from potentially very long distance effects, for example, classical decoherence.

The simulation presented here operates in the $S$-matrix oscillations formalism [37] and was realised in Python, but for the neutrino propagation the code gives the same results as the Fortran simulation found in [38]. In the case of quantum decoherence where the neutrino exists as a single mass eigenstate, the neutrino is not coherent and the Earth matter effect causes no significant change to the measured flavor composition at low energies. For the case of propagational decoherence, the neutrino is a decoherent superposition of mass states where the description of the neutrino in the flavor basis is given by constant phase differences between the mass eigenstates and an effect may be seen. While many models for supernova neutrino spectra, see, for example, [39, 40], and other astrophysical neutrino sources may be interesting, for simplicity, we adopt an initial uniform electron neutrino flux with energies between 0.5 and $20 \mathrm{MeV}$. We are interested in a significant measurement so incorporated propagation of the neutrino directly through the Earth and have obtained a maximal difference between propagation decoherence and the quantum decoherence effect at greater than $100 \%$. The latter is important for next generation neutrino measurements.

2.5. Analytic Illustration: Two-Flavour Case without Core. The theory of neutrino propagation, including neutrino propagation in medium and neutrino propagation when the neutrino experiences propagation decoherence, is well presented in the papers by Beuthe [22] and Akhmedov 
and collaborators [41, 42] and Blennow and Smirnov [43]. These papers give the essential understanding of neutrino propagation in matter and propagation decoherence, but no explicit formula is given for a neutrino which undergoes propagation decoherence and then experiences the Earth matter effect.

For ease of discussion we will consider just two regimes, the vacuum and the Earth (with constant density) and two neutrino flavors. Due to the discontinuity at the Earth's surface, the adiabatic formulas do not describe the neutrino propagation. However, the solution is to match the flavor conditions between the two regimes. The flavor at the point before the density jump is used to determine the initial state after the jump $[43,44]$.

Propagation decoherence was studied in detail by Beuthe [22] but unfortunately only in the vacuum case. Akhmedov and Wilhelm [41] explicitly consider decoherence due to production or detection conditions but only note that finite coherence length is recovered during the integration over energy. Beuthe [22] goes into great detail about the physics, which is that the wave packets separate or that the wave packet spatial spread is so large that the phase varies over the wave packet and the information is lost. These are changes of the relationship between the states which make up the neutrino and the changes can be accounted for by integrating the phase in the vacuum transition probability. In the case of two regimes with a sharp transition, as we consider here, it is necessary to find the flavor states at the transition.

The condition for the wave packet separation to be complete is given explicitly by Farzan and Smirnov [24]. They explicitly note that this is different than the effect due to averaging (Section 2.1 in [24]) over the energy, despite the effect being computationally the same for the vacuum (and adiabatic) case [22]. As noted by [24], once the phase difference becomes large, the phase difference between the mass eigenstates can be expressed as a constant. This happens once [24]; consider

$$
\begin{aligned}
\sigma_{x} \ll & d_{L} \\
= & 3 \\
& \quad \times 10^{-3} \mathrm{~cm} \frac{L}{100 \mathrm{Mpc}} \frac{\Delta m^{2}}{2.5 \times 10^{-3} \mathrm{eV}^{2}}\left(\frac{10 \mathrm{TeV}}{E}\right)^{2},
\end{aligned}
$$

which is achieved for both $\Delta m_{12}^{2}<1 \mathrm{eV}$ and $\Delta m_{23}^{2}<1 \mathrm{eV}$ for $L=10 \mathrm{kpc}$ since we expect $\sigma_{x}$ to be less than $10^{-10}$.

To determine the proper state we must consider the proper normalisation and phase for the states. In the twoflavor approximation, the probability is given by

$$
\begin{aligned}
& P_{e e}=\frac{1}{2}\left(1+\cos ^{2}(2 \theta)\right), \\
& P_{e \mu}=\frac{1}{2} \sin ^{2}(2 \theta),
\end{aligned}
$$

where $\theta$ is the neutrino two-flavor mixing angle in vacuum and $e$ and $\mu$ are the two neutrino flavors. The amplitude can then be given by

$$
\begin{aligned}
& A_{e e}^{\mathrm{dec}}=\cos ^{2}(\theta) e^{i(3 \pi / 4)}+\sin ^{2}(\theta) e^{-i(3 \pi / 4)}, \\
& A_{e \mu}^{\mathrm{dec}}=\sin (\theta) \cos (\theta)\left(e^{-i(3 \pi / 4)}-e^{i(3 \pi / 4)}\right) .
\end{aligned}
$$

These give the correct flavor amplitudes of a neutrino produced in a $\nu_{e}$ state which has travelled through vacuum and experienced wave packet separation when it reaches the earth-to-vacuum transition. There is an overall phase, but for length scales (such as the earth-to-vacuum transition) much smaller than the the distance between the wave packets (which can be $1 \mathrm{~km}$ or more) the phase difference between the wave packets is a constant as expressed above. An amplitude where the phase between the wave packets changes with distance would be incorrect for large wave packet separations.

This allows us to give a clear description of a produced $v_{e}$ which experiences propagation decoherence, travels through the mantle of the earth, and then is detected as a $v_{e}$. This is

$$
P_{e e}^{\mathrm{prop}}=\left|A_{e e}^{\mathrm{dec}} A_{e e}^{\mathrm{mat}}+A_{e \mu}^{\mathrm{dec}} A_{\mu e}^{\mathrm{mat}}\right|^{2}
$$

which for the standard description in terms of a matter mixing angle $\theta_{m}$ and the matter phase $x_{m}$ is

$$
\begin{aligned}
& P_{e e}^{\text {prop }}=\frac{1}{8}\left(2 \cos ^{2}\left(x_{m}\right)(3+\cos (4 \theta))+\sin ^{2}\left(x_{m}\right)(4\right. \\
& +\cos \left(4 \theta_{m}\right)+\cos \left(4 \theta_{m}-8 \theta\right)+2 \cos \left(4 \theta_{m}-4 \theta\right) \\
& \left.\left.\quad+\sqrt{8} \sin ^{2}(\theta) \sqrt{3+\cos (4 \theta)} \sin \left(4 \theta_{m}-4 \theta\right)\right)\right)
\end{aligned}
$$

where [37]

$$
\begin{array}{r}
\sin \left(2 \theta_{m}\right)^{2}=\frac{\sin (2 \theta)^{2}}{\sin (2 \theta)^{2}+\left(\cos (2 \theta)-2 A E_{v} / \Delta m^{2}\right)^{2}}, \\
x_{m}=x \sqrt{\sin (2 \theta)^{2}+\left(\cos (2 \theta)-2 A E_{\nu} / \Delta m^{2}\right)^{2}}, \\
x=\frac{\Delta m^{2} L}{4 E_{v}},
\end{array}
$$

and $A$ is a constant density. This formula is different from that which is given for solar neutrinos and which is presented in the paper by Dighe et al. [45]. They give the calculation for an incoherent mixture of mass eigenstates originating in the sun, but this important paper does not explicitly consider propagation decoherence (decoherence due to wave packet separation) but rather the effects of coherent neutrino propagation at long baselines in addition to the known solar MSW resonance effect where neutrinos which leave the sun exist in only a single mass eigenstate. Farzan and Smirnov [24] explicitly give the condition to have the phase change for decoherent, and also for detection to restore coherence. The condition is that the measurement takes place over large enough time scales (or small enough energy resolutions) for both wave packets to be measurable [46]. 
In the case of quantum decoherence we have the emission of a graviton off a neutrino mass state in the vacuum. An interaction of a neutrino with the graviton serves essentially as a measurement of the neutrino state, both its detection and production in quantum mechanical language. This tells us the condition on the graviton which must be true for the effect presented in this work; it is the condition of coherent production/detection of a neutrino as presented in [22, 41]. The formulation can be considered in the framework of [41] but where the $\widetilde{U}$ matrix elements represent the mass eigenstates and not the matter eigenstates (so the identity since we are assuming propagation in the vacuum). Then the relationship for neutrinos which have undergone quantum gravity decoherence is simply (for two flavours)

$$
P_{\alpha \beta}=P_{\alpha 1} P_{1 \beta}^{\text {earth }}+P_{\alpha 2} P_{2 \beta}^{\text {earth }} \text {. }
$$

This is equivalent, as discussed in [45], to a coherent neutrino being observed a long distance from the neutrino source by an experiment without arbitrary energy resolution. The probability is then given by

$$
\begin{aligned}
& P_{e e}^{\text {grav }}=\frac{1}{16}\left(10+2 \cos \left(4 \theta_{m}\right)-\cos \left(4 \theta_{m}-2 x_{m}\right)\right. \\
& \quad+2 \cos \left(2 x_{m}\right)-\cos \left(4 \theta_{m}+2 x_{m}\right) \\
& \quad+4 \cos (4 \theta)\left(\cos ^{2}\left(2 \theta_{m}\right)+\cos \left(2 x_{m}\right) \sin ^{2}\left(2 \theta_{m}\right)\right) \\
& \left.\quad+4 \sin \left(4 \theta_{m}\right) \sin ^{2}\left(x_{m}\right) \sin (4 \theta)\right),
\end{aligned}
$$

where $x_{m}$ and $\theta_{m}$ are as mentioned before.

The ratio of neutrinos which have undergone propagation decoherence and at the same time propagated through a region of constant density to those which have only propagated through the vacuum is given by the following expression:

$$
\begin{aligned}
& R_{p} \\
& =\frac{\left(\cos \left(x_{m}\right)^{2}(3+\cos (4 \theta))+\left(2+\cos \left(4 \theta_{m}-8 \theta\right)+\cos \left(4 \theta_{m}-4 \theta\right)\right) \sin \left(x_{m}\right)^{2}-2 \sin \left(2 x_{m}\right) \sin \left(2 \theta_{m}-2 \theta\right) \sin (2 \theta)\right)}{(3+\cos (4 \theta))} .
\end{aligned}
$$

Analogically, the ratio of neutrinos which have undergone quantum decoherence in the presence of matter effect in a constant density medium to those which have propagated through the vacuum takes a different form:

$$
R_{q}=\frac{5+\cos \left(4 \theta_{m}\right)+\cos \left(4 \theta_{m}-4 \theta\right)+\cos (4 \theta)+4 \cos \left(2 x_{m}\right) \cos (2 \theta) \sin \left(2 \theta_{m}-2 \theta\right)}{6+2 \cos (4 \theta)} .
$$

A difference between the ratios $R_{p}$ and $R_{q}$, in principle, could be measurable and indicates the principal difference between propagation and quantum decoherence emerging in the presence of matter effect. Measurement of such a difference could therefore serve as a clear example of graviton detection.

\section{Graviton-Neutrino Scattering}

Now consider which quantum gravity processes the neutrino could possibly experience so as to experience the quantum decoherence effect in the astrophysical medium. As mentioned we will be considering quasi-classical gravity processes.

As is known the Coulomb field is measured by inserting a charged probe into it. From the quantum electrodynamics (QED) point of view, an electromagnetic scattering of a charged particle off the Coulomb field is due to an exchange of virtual photons (with small negative momentum transfer squared $-q^{2}=Q^{2}>0$ in the $t$-channel) between the probe and the source. Analogically, it is correct to discuss multiple exchange of virtual $t$-channel gravitons in a scattering event as a signature of non-zeroth curvature itself (for more detailed discussions of the principles, see, for example, [47]).

Generically, in quantum electrodynamics (QED) the virtual photons may become real (produced on mass-shell) if one disturbs the field pumping energy into it. This is the physical reason for photon Bremsstrahlung in QED. Specifically, the standard Bethe-Heitler scattering in electrodynamics demonstrates that only an accelerated charge emits real photons (corresponding to electromagnetic wave in the classical limit of multiple soft photon radiation). Likewise, in the quasi-classical gravity framework, the virtual graviton, as a quantum of the gravitational field of a static massive object, may turn into the real one (corresponding to gravitational wave in the classical limit of multiple soft graviton radiation) if the source of the gravitational field is accelerated or, in general, when the energy-momentum tensor experiences disturbances.

Possible sources of real gravitons in the universe include active galactic nuclei (AGN), binary systems, supernova explosions ( $\mathrm{SNe}$ ), primordial black holes collisions, compact star/black holes binaries, quantum bremsstrahlung of gravitons of particles scattering off a massive object, black hole 
(BH) evaporation, relic isotropic gravitational background from the early universe, inflation, phase transitions in the primordial plasma, and the decay or interaction of topological defects (e.g., cosmic strings). For details and references, see [48].

Consequently, in the cosmological medium a neutrino can scatter either off a classical gravitational potential with accompanying radiation of an energetic real graviton off the scattered neutrino (e.g., Bethe-Heitler-type scattering) or off real graviton in the astrophysical medium (e.g., Comptontype scattering). Let us consider both cases and conditions for initiation of the quantum neutrino decoherence in more detail.

3.1. Gravitational Bethe-Heitler Scattering. In fact, all elementary particles, including neutrinos, when traveling in the vicinity of massive objects (sources of classical gravitational field) can emit real gravitons with a certain energy spectrum. This process has a straightforward QED analog of a photon emission in relativistic electron scattering off the Coulomb field of a heavy nucleus mentioned above, the Bethe-Heitler process at the Born level. Even though the energy spectrum of radiated real gravitons is peaked in the forward direction and in the infrared limit (corresponding to forward radiation of classical gravitational waves), there is a nonnegligible probability to radiate hard or energetic gravitons, namely, with energies comparable to the incoming relativistic neutrino energy. Due to the quantum nature of the neutrino and graviton, the latter process can trigger a dramatic decoherence of an incoming neutrino flavor state at the quantum level during a very short time scale (inversely proportional to the energy of the radiated hard graviton).

The decoherence of the neutrino at the quantum level can only be initiated by hard energetic interactions with relatively hard gravitons whose energies exceed the mass difference between different mass states $E_{G} \gtrsim \Delta m_{i j}$ and therefore requires hard real graviton emission. In this case, the hard graviton probe has a small wave length and thus can resolve separate mass states in a coherent or incoherent neutrino flavor state in the quantum mechanical sense (likewise a hard enough photon can resolve an internal substructure of the proton wave function and interacts with the separate quarks it is composed of while a soft one "sees" a proton as a whole only).

A soft graviton with energy lower than the difference between mass states will be unable to resolve the individual mass eigenstates in this superposition and will instead couple to the whole energy-momentum tensor of the flavor state, nonlocally, which is the classical general relativity limit. In the latter case quantum decoherence is not triggered, and the effect will be as discussed in [31].

The Born-level calculation is good first order approximation in the case of off-forward hard graviton emissions at large angles relevant for the quantum decoherence effect; this is the reason why one can disregard higher-order radiative corrections which are highly suppressed (by extra powers of the Planck mass) as long as one cuts off the problematic but uninteresting infrared/collinear parts of phase space. As was previously shown in [49], the radiative corrections can only be relevant in the deep infrared limit of soft real gravitons $E_{G} \rightarrow 0$ emitted in the forward direction where they will cancel the soft/collinear divergences. The latter classical limit represents classical gravitational waves emitted off a neutrino state with very small or no impact on it.

In the considered GBH case, shown in Figure 1(a), one deals with the graviton exchange with negative momentum transfer squared $t=-q^{2}<0$ in the $t$-channel with the propagator stretched between the relativistic neutrino of mass $m_{\nu}$ and energy $E_{\nu} \gg m_{\nu}$ and a massive classical gravitational field source with mass $M \gg E_{\gamma}$. The wave function, $\Psi_{v_{f} \rightarrow v_{a}}$, describes a projection of a given flavor state $f$ onto a fixed mass state $a$ denoted as a dark ellipse, while the heavy classical source of the gravitational field is shown by a shaded circle.

The GBH cross section has initially been calculated for the gravitational scattering of scalar particles with $M \gg$ $m$ in [49]. In the soft graviton limit, the graviton-neutrino coupling is not sensitive to the spin of an incident relativistic particle to leading order, while the classical nonrelativistic source can be considered spineless in this first discussion for simplicity (in principle, helicity dependence of hard gravitonneutrino interactions can be a relevant topic for further studies). We therefore use their formula as a sufficiently good approximation to estimate the neutrino-solar mass cross section with graviton radiation numerically. In this case, as an order-of-magnitude estimate, the GBH cross section at the Born level behaves as

$$
\sigma_{\mathrm{GBH}} \sim \frac{M^{2} E_{v}^{2}}{M_{\mathrm{Pl}}^{6}}, \quad M \gg E_{\nu} \gg m_{v}
$$

and thus may not always be very small since the Planck scale suppression can be largely eliminated by having a mass $M$ of a heavy classical source in numerator. In particular, for a solar mass object $M \sim 10^{57} \mathrm{GeV}$, we have $M^{2} / M_{\mathrm{Pl}}^{6} \sim 1 \mathrm{GeV}^{-4}$, so there is no significant suppression of the cross section for relativistic neutrinos. This is a particle physics magnitude cross section which naively implies particle physics size impact parameters. Larger impact parameters would exist for larger masses, such as the dark matter halo. The above cross section is integrated over impact parameter, and it may be instructive to look into differential cross section in order to find the probability of this process as a function of distance to the massive astrophysical body. For the current study it suffices to note that such probability can potentially be significant.

It is worth noticing that the Bethe-Heitler calculation in QED to first order gives the correct cross section for the photon Bremsstrahlung for extended objects such as a nucleus as shown in [50] ([51] demonstrates that after advances it is still correct to first order in the hard photon limit). Similarly, one may expect that the GBH result for a point-like classical source of gravitational potential should be roughly correct to first order for extended objects as well, like a star or even a dark matter distribution in the quasi-classical gravity case. The QED calculations are provided in the above references for scattering due to the field surrounding the nucleus; the nucleus is small compared to the total size of the field and scattering off the nucleus via other processes can be 


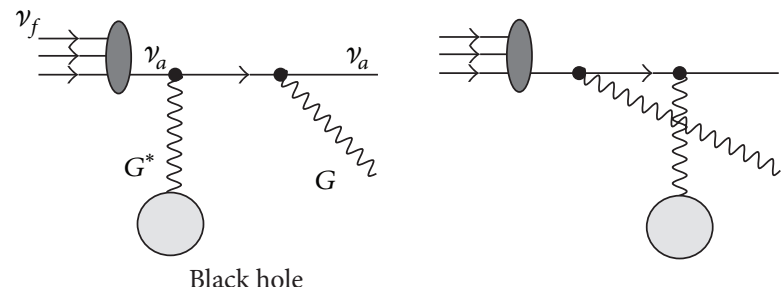

(a)
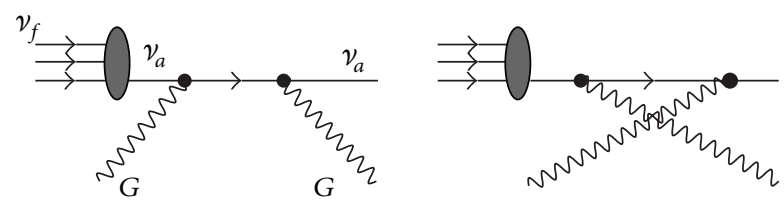

(b)

FIGURE 1: The quasi-classical gravity processes which destroy the coherence of the neutrino flavor eigenstate $(f=e, \mu, \tau)$ at the quantum level effectively turning it to a mass eigenstate $(a=1,2,3)$, the gravitational Bethe-Heitler-type scattering of neutrino off a massive object, for example, a black hole (a) and the gravitational Compton scattering (b). The dark ellipse is a projection to a fixed mass state and the shaded circle is a classical source of the gravitational field.

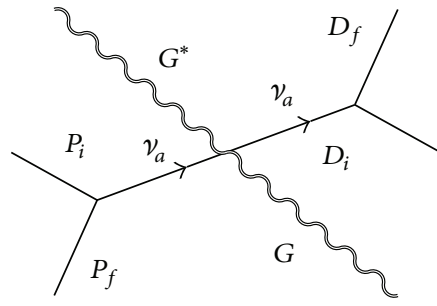

(a) Diagram for neutrino propagation with quantum decoherence

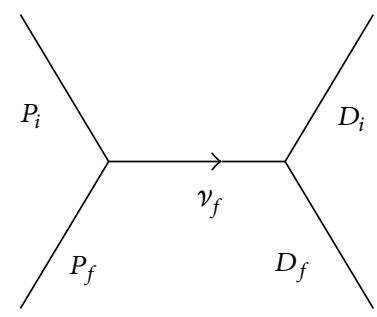

(b) Diagram for neutrino propagation
Figure 2: Shown are diagrams of neutrino propagation in the quantum field theoretical description (such as found in [22]). The neutrino is described as a stretched propagator between the production $(P)$ and detection $(D)(b)$ weak processes (with initial and final states denoted by subscripts $i$ and $f$ ). In the case of quantum decoherence (a), the emission of a graviton in between the production and detection processes means that the neutrino exists in a definitive mass state since both the propagation Hamiltonian and graviton interaction Hamiltonian conserve mass. The quantum decoherence effect described here follows from the discussion in Section 2 of [22].

safely ignored. Most of the cross section is thus not due to trajectories where the electron passes through the nucleus. We expect that this is also true for the GBH scattering, where most of the flux which scatters off the star or other massive object will not pass through the star, and the pointlike estimation (15) remains valid.

In Figure 3 we have presented the differential (in radiated graviton energy $E_{G}$ and neutrino angle $\theta_{\nu}$ ) and integrated cross sections of the $\mathrm{GBH}$ process for typical $\mathrm{MeV}$-scale astrophysical neutrinos and a solar mass scale source of the gravitational field. As expected, the main bulk of the cross section comes from the soft gravitons (gravitational waves) emission in the forward limit. It is remarkably important, however, that there is a long nonnegligible tail in the differential distributions of the GBH cross section in the single real graviton energy $E_{G}$ and emission angle $\theta$. It turns out that such a tail to harder/off-forward gravitons is not very strongly suppressed; typical GBH scattering cross sections for SNe neutrino energies of $E_{v} \sim 10-100 \mathrm{MeV}$ and a solarmass classical source are found to be around $\sigma \sim 0.1-10$ millibarns, which are some 16-18 orders of magnitude larger than typical neutrino-electron scattering cross sections (less than an attobarn at the same energies).

This observation strongly suggesting the importance of the quantum decoherence initiated by interactions with such energetic real gravitons. The latter source of decoherence does not have a classical interpretation. As we have already mentioned, due to a quantum mechanical nature of a single hard graviton emission at energies $E_{G} \gtrsim \Delta m_{i j}$ (with local coupling to a gravitational mass eigenstate) and universal quantum mechanical time-energy uncertainty arguments, the considered effect of neutrino flavor decoherence is a purely quantum effect. The hard Gravi-strahlung effect is thus relevant for a broad range of neutrino energies, and one can utilize the SNe neutrinos as a clear sample since (1) fluxes of SNe neutrinos are the largest among astrophysical neutrinos and (2) $\mathrm{SNe}$ neutrino emission mechanisms are the best understood among other possible astrophysical sources.

Of course, the $t$-channel gravitons are extremely soft and form classical gravitational potential of a classical massive source and they do not trigger a decoherence of the neutrino state; only the hard real graviton emissions are relevant.

\subsection{Probability for Quantum Gravitational Decoherence.} The cross section of the considered $\mathrm{GBH}$ process can be enhanced for the galactic center $\left(\sim 10^{6}-10^{9}\right.$ solar masses $)$ or the dark matter halo $\left(\sim 10^{20}-10^{24}\right.$ solar masses $)$. It can also be enhanced for ultra-relativistic neutrinos which are potentially detectable at neutrino observatories such as IceCube and Super-K. As is our main result, we notice that the GBH scattering may cause the quantum decoherence of astrophysical neutrinos and this effect can be measured via neutrino flavor composition measurements. A massive classical source of the gravitational field may not necessarily be a black hole, but any compact star or, in general, any bound gravitational potential induced by continuous matter distribution in the galactic disk and halo.

Due to rather large cross sections it can be that most of the astrophysical neutrinos which are observed at the Earth from a given direction and have passed in close vicinity of a massive object would have experienced the quantum decoherence due to a graviton-induced scattering. In other words, the probability for a given neutrino in a superposition of mass states $f$ to decohere, being "transformed" into one of the mass states $a=1,2$ or 3 in the GBH process, $P_{G} \sim\left|A^{(G)}\right|^{2}$, may be large for possible (massive) astrophysical sources of classical gravitational fields, depending on details of astrophysics and quasi-classical gravity. Deviations from quasi-classical gravity 


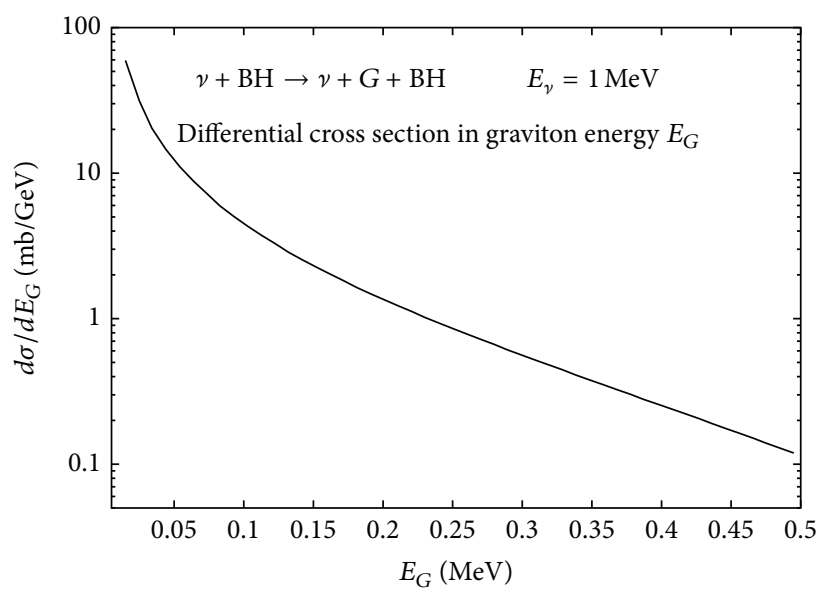

(a)

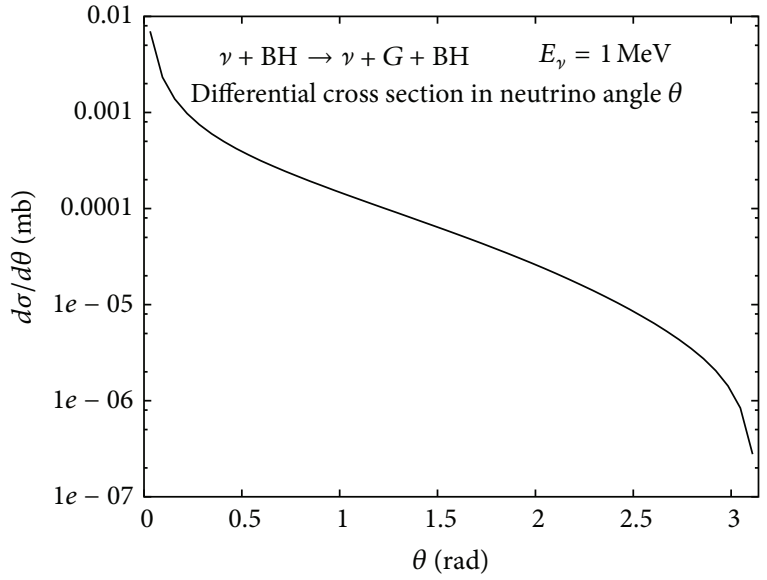

(b)

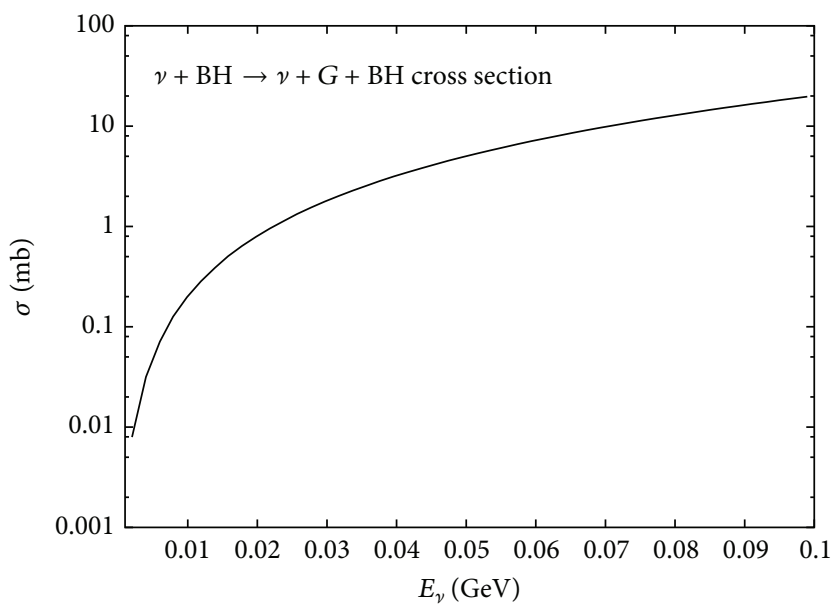

(c)

Figure 3: Differential cross section of the gravitational Bethe-Heitler scattering of neutrino off a massive object, for example, a black hole $(\mathrm{BH})$ in radiated graviton energy $E_{G}(\mathrm{a})$, in polar angle of the final-state neutrino $\theta_{v}(\mathrm{~b})$, and the integrated cross section as a function of incoming neutrino energy $E_{\gamma}$ typical for astrophysical sources, for example, see [69], (c).

which illuminate the fundamental quantum gravity theory may also be relevant. To parameterise this we can define

$$
P_{G} \equiv \frac{N_{G v}}{N_{\text {init }}},
$$

where $N_{G v}$ is the number of neutrinos which have radiated off an energetic graviton with $E_{G} \gtrsim \Delta m_{i j}$ while being scattered off a massive object (on the way to the Earth, a produced mass state may experience more graviton-induced rescatterings in classical gravitational potentials which do not affect the coherence of the neutrino state any longer but may cause an additional energy loss of the propagating neutrino into the gravitational radiation) and $N_{\text {init }}$ is the total number of neutrinos which have been emitted off an astrophysical source. As we will demonstrate below, the $P_{G}$ value can be measured via neutrino flavor composition observations leading to a promising opportunity for experimental tests of quantum gravity induced interactions.

A precise theoretical calculation for $P_{G}$ is influenced by many potentially relevant aspects. First, it depends on a quantum gravity model through model-dependent local neutrino-graviton couplings, thus offering a good opportunity for experimental tests of quantum gravity. Due to the extended nature of many of the sources, there might be strong differences for models with some nonlocality. Second, it may be influenced by yet unknown higher-order corrections and by multiple rescatterings of a neutrino off a massive source, multiple massive sources, or a diffuse source such as the dark matter halo which the neutrino passes through on its path to the Earth (in this case, the Eikonal approximation for neutrino-graviton rescattering can be used [52]). Thus, the actual cross sections may significantly vary depending on environment a neutrino propagates in. Thirdly, the astrophysical neutrino flavor composition may depend on production processes which may currently be unknown. Also, energy loss of the neutrino due to the hard Gravi-strahlung in each scattering event should be taken into consideration, together with other effects which change the coherence of the neutrino state. This could also be used to identify a graviton interaction, if, for example, this lower energy flux comes 
some short time later than the initial flux. Finally, including possible dense astrophysical media might be important as the neutrino may have additional weak rescatterings off normal matter acting on the neutrino leaving the neutrino in a superposition of mass eigenstates when it arrives at the Earth. Therefore, additional astrophysical information is desired to constrain these uncertainties. All of the above aspects are the major unknowns in making predictions for the $P_{G}$ quantity which require a further effort of the quantum gravity, neutrino, and astrophysics communities.

3.3. Gravitational Compton Scattering. Another possibility for quasi-classical gravity induced interactions with neutrino participation is shown in Figure 1(b). This is the (tree level) gravitational Compton scattering of a neutrino off a real graviton in cosmological medium. The latter process has been previously studied in [53] and in many other papers. The cross section in this case is always extremely small $\sigma \sim E_{\gamma}^{2} / M_{\mathrm{Pl}}^{2}$ for a $\mathrm{MeV}$ neutrino, and real graviton fluxes are not expected to compensate for such a huge suppression. This process seems less interesting when applied to astrophysical neutrino flavor composition. Hypothetically, this effect could be considered in exotic cases of ultra-relativistic neutrinos and/or in the very early universe where the graviton fluxes might have been rather intense.

\section{Quasi-Classical Gravity Measurement Proposal}

As presented above, the neutrino in a mass eigenstate does not oscillate unless it scatters off ordinary matter via a weak channel which will cause it to be in a flavor eigenstate. It is likely that the $Z, W$-mediated scattering happens only in the Earth-based detector enabling us to access information about the graviton-neutrino scattering which might have happened far away from the solar system. In the considered situation, the neutrino plays an analogical role of an electric charge in a quantum measurement of the microscopic Coulomb field properties. From the quantum mechanical point of view, a massive object (e.g., dark matter distribution or the galactic center) vicinity can then be viewed as a macroscopic "detector" of gravitons. The neutrino scattering off a massive object and radiating an energetic graviton by means of the local graviton-neutrino coupling would be an elementary act of quantum mechanical measurement, and the neutrino conveys the quantum information about the act of graviton measurement to the Earth (see Figure 2). The neutrino does not undergo oscillation or demonstrate properties consistent with being a superposition of mass eigenstates since it is in a definitive mass state during the propagation and graviton interaction. The neutrino not interacting weakly as it travels is a good approximation due to extremely weak interactions of neutrinos with ordinary matter. Then an Earth-based detector will "read off" the results of the "graviton measurement" which has taken place at the massive object.

Previously, in [5], it has been claimed that it is not possible to detect a single graviton with a planet-scale detector. Our proposal is to measure the described graviton-neutrino scattering effect (specifically, the gravitational Bethe-Heitler scattering of neutrino off a massive object) experimentally, which is the best possibility for indirect graviton detection proposed. Remarkably, we consider a super massive-scale "detector" of energetic gravitons, with neutrinos serving as the most efficient carrier of the information about such a measurement to the Earth.

\subsection{Quantum Gravitational Decoherence Effect on Neutrino} Oscillations. Here we consider a very massive source of strong gravitational fields like cluster of stars (e.g., the center of our Galaxy) or a dark matter halo as a good example of a graviton "detector." This section provides predictions for such an extreme large-scale quasi-classical gravity measurement.

As we have demonstrated above, the probability of an individual (elementary) act of the "quantum gravity measurement" defined by the graviton-neutrino cross section can be rather large due to a large $\mathrm{GBH}$ cross section and there may be scenarios where it should not be neglected.In particular, utilizing the dense region of stars and black holes in the galactic center (GC) as our "graviton detector" in the above sense, one could expect that a significant fraction of neutrinos passing by the dense region would have experienced the GBH scattering. Then since many of the neutrinos are now in a mass eigenstate, they will no longer undergo flavor oscillation. Due to the neutrino existing in a mass eigenstate during propagation, further graviton rescattering would not constitute additional quantum measurements of an "undetermined" quantum state. Depending on the astrophysical process, one might favor energies of neutrinos where the neutrino oscillation may not be suppressed due to the MSW effect where the neutrino exists in a single mass eigenstate, so that the graviton-induced effect would be cleaner. We suggest that this effect could be tested in neutrino telescopes and observatories by looking at the galactic center neutrino flavor composition and comparing it to the composition expected without quantum gravitational decoherence. It might be possible that close, "standard candle," neutrino emitters in other parts of the sky provide a flux of neutrinos which have not undergone quantum gravitational decoherence (note: a very similar effect should take place in flavor oscillations in the neutral kaons $K_{l}, K_{s}$ system as well).

The general formula for the number of electron type neutrinos observed from an electron type source in the vacuum is

$$
\frac{N_{e, \text { det }}}{N_{e, \text { init }}} \propto P_{e e, \infty}^{\mathrm{vac}}\left(1-P_{G}\right)+P_{G} \sum_{i=1,2,3} V_{e i} V_{i e}^{*} V_{e i} V_{i e}^{*} .
$$

Here $P_{e e, \infty}^{\mathrm{vac}}$ is the standard vacuum oscillation probability [36] far away from the neutrino source and $P_{G}$ is the probability for neutrino in a flavor state to interact with at least one graviton (16) which will depend on the graviton-neutrino scattering cross section. Every mass eigenstate of the (relativistic) neutrino shares the same energy so $P_{G}$ takes the same value.

If all neutrinos have interacted with at least one graviton, that is, fixing $P_{G}=1$, than the expression for the total $\nu_{e} \rightarrow$ $v_{e}$ transition probability becomes

$$
P_{e e}^{G}=\cos ^{4} \theta_{12} \cos ^{4} \theta_{13}+\cos ^{4} \theta_{13} \sin ^{4} \theta_{12}+\sin ^{4} \theta_{13},
$$


where $\theta_{12}, \theta_{13}$, and $\theta_{23}$ are the standard neutrino vacuum mixing angles. This basic formula is our prediction (in vacuum) for the "maximal decoherence" scenario valid for $P_{G} \simeq 1$. In the standard Large mixing angle (LMA) global fit with $\sin ^{2} \theta_{13}=0.025, \sin ^{2} \theta_{12}=0.31$, and $\sin ^{2} \theta_{23}=0.60$ (but with $\delta_{C P}=0$ ) [54], the value for the transition probability throughout a range of neutrino energies is shown in Figure 4. The difference between the predictions for $P_{G}=1$ and $P_{G}=0$ is that a neutrino which has experienced propagation decoherence will have a constant phase difference in the flavor basis at the earth/vacuum boundary, possibly resulting in a greater than $100 \%$ change in the survival probability depending on neutrino energy, the detector resolution, and the detector location. Further detail is available in the Appendix. Here the simulation for neutrino propagation in matter and vacuum was based on [38].

As one notices in Figure 4, the relative effect ranges from a few percent at $E_{\gamma} \simeq 2 \mathrm{MeV}$ to about $350 \%$ at $E_{\gamma} \simeq 20 \mathrm{MeV}$. This is expected because the electron type component of the super position of three decoherent mass states is larger than the electron type component of the single mass states. The Earth matter effect depends on neutrino energy and so the effect will be larger at higher energy ( $20 \mathrm{MeV}$ and above) than at smaller energies $(2 \mathrm{MeV})$. This gives a quite noticeable difference between the case where the neutrino undergoes propagational decoherence relative to the case where the neutrino experienced quantum decoherence.

For illustration in this calculation we use a constant, maximal probability for quantum decoherence case, $P_{G}=1$. In practice we expect this to be less than one and to depend on the neutrino energy $E_{\gamma}$. The $P_{G}\left(E_{v}\right)$ value can be considered as an observable and extracted from the flavor composition data and further compared to theoretical calculations. Possible sources of neutrinos in extreme astrophysical environments include the aforementioned dense galactic center, but also ordinary stars, $\mathrm{SNe}, \mathrm{GRB}, \mathrm{AGN}$, and other galactic or extragalactic sources $[55,56]$. Quantum gravity models should aim at predicting the $P_{G}$ in these extreme environments as a function of astrophysical parameters and neutrino energy so that favored models can be constrained by the neutrino flavor data.

The neutrino flux spectrum from astrophysical sources is still being modeled [57-59]. By comparing observed neutrino flavor composition for neutrinos passing through the Earth to that of neutrinos which have not passed through the Earth, the flux can be divided out and a possibly large effect may be observed (for the above LMA global fit). This could be visible in flavor data at large statistics.

This is our prediction for the quantum gravity-induced effect on the detected flavor composition in the "maximal quantum decoherence" scenario. While numerically the effect can be very large, one would certainly need to have a good understanding of all the other statistical and systematical uncertainties for a possible measurement of the dependence of $P_{G}$ on neutrino energy. Current generation neutrino observatories can observe approximately ten thousand total events from nearby ( $10 \mathrm{kpc}) \mathrm{SNe}$; however, next generation neutrino observatories (such as Hyper-K) can provide statistics to reduce systematical uncertainties [60]. Additionally,

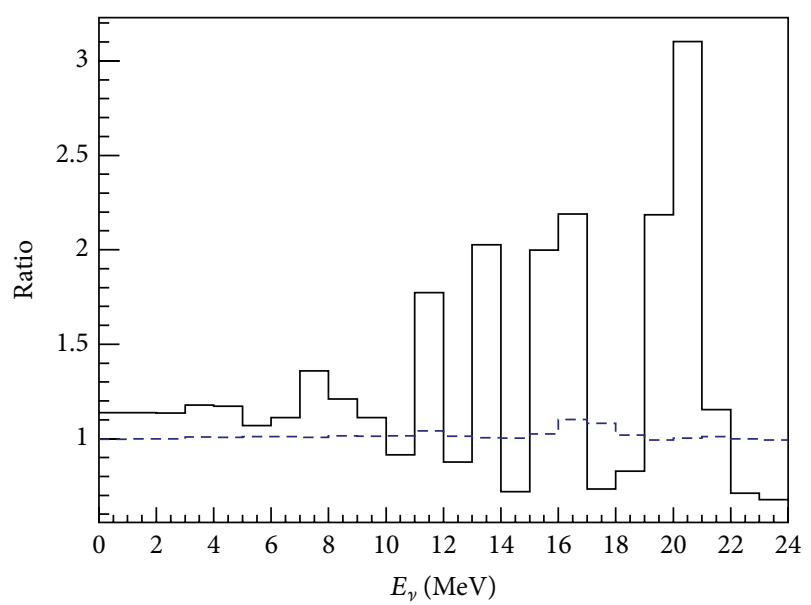

FIGURE 4: Here the difference due to the Earth matter effect in the observed number of electrons is plotted (assuming an initial flux that only contains electron neutrinos). The difference is defined to be the ratio of the observed number of electron type neutrinos for the case where there is a maximal Earth matter effect (impact parameter is $\sin (.576))$ and the case where there is no earth matter effect. Shown are two curves, black solid for $P_{G}=0$ (propagation decoherence case) and blue dotted for the $P_{G}=1$ (quantum decoherence case). This calculation was done with a uniform distribution of decoherent electron neutrinos. The difference due to the Earth matter effect may be higher than $300 \%$. In the case of fixed $P_{G}=1$, the flavor composition of the neutrino flux is minimally affected by the Earth at low energies.

improvements are needed in current neutrino flavor reconstruction technologies, which can reconstruct the neutrino flavor to the percentage level [61]. It has been pointed out that sensitive neutrino detectors should be placed in North America (Sudbury), Japan (Hyper-K), Chile (ANDES), and Antarctica (Beyond DeepCore) to best observe SNe neutrinos and the effect that the Earth has on SNe neutrinos [62].

\section{Conclusion}

In conclusion, we have considered a quasi-classical gravity process, the gravitational Bethe-Heitler scattering of a neutrino off a massive object accompanied by an energetic graviton radiation, which can have a rather large cross section proportional to the mass squared of the classical source. Due to hard gravitons interacting with a neutrino mass eigenstate only, opposite to weak bosons which interact with a flavor eigenstate only, the considered process is a measurement of the incoming neutrino flavor state at the quantum level, causing its decoherence in a different manner than the process which can be called propagational decoherence or other sources of decoherence. This quantum decoherence affects astrophysical neutrino behavior. Namely, quantum decoherence can be considered as a specific quasi-classical gravitational measurement of the neutrino propagating state, which changes the behavior of the neutrino in the presence of a potential (such as the Earth matter effect) compared to the traditional source of decoherence known as propagation 
decoherence, which can be observed in the neutrino flavor composition in an Earth based detector (see the Appendix).

This enables the utilization of neutrinos traveling across the Galaxy as a source of information about the gravitoninduced interactions they might have experienced on their journey to Earth. Specifically, the measured probability to find a given flavor component in the neutrino flux coming from a vicinity of a super massive black hole or another super massive object (galactic center or dark matter halo) will be different from the corresponding probability measured from a source of neutrinos where the neutrinos never pass near a massive system. In the case where no astrophysical neutrinos can be identified which have not interacted with a gravitational potential, the flavor composition can be compared to the expectation for the Earth matter effect which can be determined using reactor, atmospheric, and accelerator neutrinos. We have explicitly demonstrated that the maximal difference corresponding to an assumption that all of the detected neutrinos have experienced an interaction with a graviton, that is, $P_{G}=1$, is large and can be measurable at high statistics. Assuming that the required astrophysical conditions are met for large $P_{G}$, this would provide a first measurement of quasi-classical gravity. Further discrimination of quantum gravity models would require more statistics and detailed calculations using these models. Additionally, we note that the energetic graviton bremsstrahlung would cause a significant decrease in energy of the neutrinos which are scattered at large angles. Since the galactic center is not only massive but is a source of neutrinos, it might be possible that a large enough $\mathrm{SNe}$ in the galactic center would produce enough neutrinos so that the existence of graviton bremsstrahlung could be induced by a group of neutrinos arriving a short time after the initial group with a lower median energy. This could be used in addition to the flavor to investigate quantum gravity models.

Thus, the probability for a neutrino state to interact with at least one energetic graviton, $P_{G}$, is considered to be a new observable containing information about the quantum gravity scattering process. An estimate of the $P_{G}$ value from neutrino flavor composition data with good angular resolution would provide an important experimental test for quantum gravity models. This is the major proposal we make in our paper. We do not expect $P_{G} \sim 1$ in most scenarios and a realistic theoretical estimate for $P_{G}$ depends on many factors and is not well-constrained yet. For a distant enough source, there are many potential scatterers which may provide the maximal case of $P_{G}=1$.

The difference between propagation decoherence and quantum gravitational decoherence is a crucial component in our study and so we provide short summary. In the classical case [22] the neutrino is produced and detected in distinct flavor states (at the astrophysical source and the earth detector) and exists as a quantum mechanical superposition of mass states due to the mass states being indistinguishable to the detection process. If the neutrino passes near a massive object, then it might undergo what we described as classical decoherence $[25,27-30]$. The different mass states continue to exist and make up the neutrino even if they cease to overlap due to what we describe as propagation decoherence [22].
In the quasi-classical gravity case (this study), the neutrino is produced and detected in distinct flavor (at the astrophysical source and the earth detector); however, the neutrino exists in a single mass state due to being "observed" by the emitted graviton which distinguishes which mass state the neutrino exists in. We describe this effect as quantum decoherence. Since only a single mass state exists, the demonstrated phenomena are different such as that which is described by the Earth matter effect where the electron type component of the neutrino experiences the electromagnetic potential of the Earth differently than the other components. For low energy neutrinos in a single mass state, the Earth matter effect is less than 1\%; in contrast to the Earth matter effect for a decoherent (due to propagation) superposition of mass states which have a stronger electron type neutrino component and experience a stronger Earth matter effect depending on neutrino energy.

While we give an explicit calculation of the GBH process to demonstrate that the emission of a hard graviton via gravitational Bremsstrahlung is relatively large and used this fact to motivate discussion of a maximal possible signature, that is, $P_{G}=1$, we expect the calculation of gravitational Bremsstrahlung to require corrections similar to that of photon Bremsstrahlung $[50,51]$ for an extended source in addition to the loop-induced corrections for a full theory of quantum gravity. Additionally, in many considered astrophysical scenarios, the astrophysical distances involved would cause $P_{G}$ to be small. This requires further work.

Having all that in mind, as a natural starting point in this very first paper we would like to present the basic concept/idea of quantum decoherence due to large angle neutrino-graviton interactions (Gravi-strahlung) in strong gravitational fields and its possible effect on neutrino flavor observables. In this paper we report on our preliminary study of such a graviton-induced effect on neutrino oscillations and motivate future studies in this direction. We plan to improve our simulation with fluxes and the astrophysical medium in a future study. The possibility that $P_{G}$ is not zero in the vicinity of the Sun should be considered as well. For example, using the same simulation as used to produce Figure 4 we find a preliminary effect for solar neutrino of approximately $3 \%$ for $P_{G}=0$ in the integrated $B_{8}$ spectrum while we see an asymmetry of $\sim 0 \%$ for $P_{G}=1$. Explicitly, the length and energy dependence of neutrino flavor oscillation will depend on the relative strengths of the graviton-neutrino interactions, the matter properties, and the vacuum oscillation parameters. Inclusion of these possibilities in the global neutrino oscillation parameter fit will be left for a later paper. Additionally, extragalactic neutrinos should be considered with additional care as GBH scattering of the neutrino off the diffuse dark matter halo may play a role. Finally, the issue of coherent production of neutrinos is not considered in this study and should be studied in detail in a future work.

The program used to produce Figure 4 can be found on the arxiv [63]. For further information about semiclassical quantum gravity see [64] and for further information about graviton bremsstrahlung see [65-67].

In the review process, [68] was brought to the attention of the authors. Here the idea that a neutrino in a superposition 
of mass eigenstates may be projected to a single mass eigenstate by a gravitational couplings was presented.

\section{Appendix}

\section{Note on Measuring Graviton Induced Decoherence}

The central effect of the graviton observation of the neutrino which is utilised in this proposed measurement is that a superposition is different than a classical ensemble of states. Distinguishing these two things is of key interest to the Quantum Information and Quantum Foundations communities and they have been shown to be different in experiments which investigate Bell Inequalities. To quote a member of the Quantum Foundations community who are also interested in distinguishing the situation where the particle is in a superposition (neutrino which has not undergone an interaction with a graviton, in our case) and those where the wave function has collapsed (in the mass basis in our case, where the neutrino has undergone an interaction with a graviton) [70]:

\section{It is a well-known and important property of quantum mechanics that a superposition of states is fundamentally different from a classical ensem- ble of states, where the system actually is in only one of the states but we simply do not know in which (this is often referred to as an ignorance- interpretable, or proper ensemble).}

What is required to distinguish these two cases is for the phase between the states to not be rapidly varying. In the case where interference phenomena may be observed (the phenomena of neutrino oscillation for neutrinos) this is obviously the case. For the case where the particle is still coherent but the phase difference between states is rapidly varying, it is obvious that it is impossible to differentiate a classical ensemble from a superposition. For neutrinos this is the situation where there is still overlap between the states but the energy resolution of the detector is not good enough to observe the oscillation and has been talked about in $[22,24]$.

However, there is an additional case where the neutrino in the flavor basis ceases to oscillate. The states no longer overlap. This is the case of propagation decoherence and generally the case for astrophysical neutrinos. In this case, in the flavor basis, the neutrino has a constant phase difference between (matter) states. If we measure this state without making any changes to it based on the phase difference we get the same result as if we measure a classical ensemble of states (the quantum gravity decoherence case). However, if we modify this (flavor) state by sending it through matter, the constant phase difference is changed differently than the classical ensemble of states and the neutrino can be distinguished as being in a classical ensemble of states (or having undergone quantum gravity decoherence) rather than a separated superposition. The boundary between matter regimes has a finite width and so the (flavor) state is going to have a constant phase difference (for large enough separations), independent of the energy resolution of the final (flavor) detector which collapses the wave function.

This can be clearly described in the density matrix formalism. In the formalism, the evolution of the density matrix is given by

$$
\dot{\rho}=-i[H, \rho],
$$

where $\rho$ is the density matrix and $H$ is the Hamiltonian. It has been shown [71] that if $\dot{\rho}=0$, knowledge about the particulars of the wave packet is unnecessary and as a consequence you can not distinguish wave packet separation from the case where you have a measurement at large distances (or you have a graviton interaction leaving the neutrino in a distinct mass eigenstate). However, we are considering the case where wave packet separation has occurred and then the neutrino passes through jump in the potential. We can describe change of basis from flavor to vacuum as $C_{1}$ and the change of basis from flavor to matter as $C_{2}$. We can describe the decoherence process as $D$, which nullifies the off diagonal components of the density matrix (e.g., [72]). Note that $D$ does not commute with $C$. We can also describe the adiabatic process of the neutrino passing through the earth as $H_{m}$. We then consider

$$
\left[C_{2}^{-1} H_{m} C_{2} C_{1}^{-1} D C_{1}, \rho\right]
$$

and note that it is not 0 . Thus $\dot{\rho} \neq 0$ and wave packet information is relevant and furthermore, as presented in this study, you may distinguish wave packet separation from graviton induced decoherence.

For the case of 2 neutrinos flavours, the constant phase difference for a neutrino in a separated superposition is obvious and is given by $\pi / 2$. For the case of 3 neutrino flavours, it is more difficult, and by brute force phase differences which work for the neutrino parameters used in this study are $0.756253 i$ and $1.477224 i$, the results of which are plotted in Figure 4 .

\section{Conflict of Interests}

The authors declare that there is no conflict of interests regarding the publication of this paper.

\section{Acknowledgments}

Stimulating discussions and helpful correspondence with Sabine Hossenfelder and Alexei Vladimirov are gratefully acknowledged. Jonathan Miller was supported in part by PROYECTO BASAL FB 0821 CCTVal and Fondecyt (Grant no. 11130133). Roman Pasechnik was supported in part by Fondecyt (Grant no. 1090291) and the Crafoord Foundation (Grant no. 20120520). Roman Pasechnik is grateful to the "Beyond the LHC" Program at Nordita (Stockholm) for support and hospitality during a portion of this work. This research was supported in part by the National Science Foundation under Grant no. NSF PHY11-25915. 


\section{References}

[1] J. Christian, "Why the quantum must yield to gravity," in Physics Meets Philosophy at the Planck Scale, C. Callender and N. Huggett, Eds., Cambridge University Press, Cambridge, UK, 2001.

[2] R. P. Woodard, "How far are we from the quantum theory of gravity?" Reports on Progress in Physics, vol. 72, no. 12, Article ID 126002, 2009.

[3] F. Dyson, "The world on a string," in The Fabric of the Cosmos: Space, Time, and the Texture of Reality, B. Greene, Ed., vol. 51, no. 8, Review of Books, New York, NY, USA, 2004.

[4] F. Dyson, "Is a graviton detectable?" in Proceedings of the Poincare Prize Lecture International Congress of Mathematical Physics, Aalborg, Denmark, August 2012.

[5] T. Rothman and S. Boughn, "Can gravitons be detected?" Foundations of Physics, vol. 36, no. 12, pp. 1801-1825, 2006.

[6] G. Amelino-Camelia, "Introduction to quantum-gravity phenomenology," in Planck Scale Effects in Astrophysics and Cosmology, vol. 669 of Lecture Notes in Physics, pp. 59-100, Springer, Berlin, Germany, 2005.

[7] T. Damour and M. Lilley, "String theory, gravity and experiment," in Proceedings of the 87th Session of the Summer School in Theoretical Physics: String Theory and the Real World: From Particle Physics to Astrophysics, Les Houches, France, July 2007.

[8] S. Hossenfelder, "Experimental search for quantum gravity," in Classical and Quantum Gravity: Theory, Analysis and Applications, V. R. Frignanni, Ed., chapter 5, Nova Publishers, 2011.

[9] L. M. Krauss and F. Wilczek, "Using cosmology to establish the quantization of gravity," Physical Review D, vol. 89, Article ID 047501, 2014.

[10] S. Weinberg, "Photons and gravitons in S-matrix theory: derivation of charge conservation and equality of gravitational and inertial mass," Physical Review B, vol. 135, pp. B1049-B1056, 1964.

[11] S. Weinberg, "Photons and gravitons in perturbation theory: derivation of Maxwell's and Einstein's equations," Physical Review B, vol. 138, pp. 988-1002, 1965.

[12] J. S. Díaz, "Neutrinos as probes of Lorentz invariance," Advances in High Energy Physics, vol. 2014, Article ID 962410, 11 pages, 2014.

[13] G. L. Fogli, E. Lisi, A. Marrone, and G. Scioscia, "Testing violations of special and general relativity through the energy dependence of $\nu_{\mu} \leftrightarrow \nu_{\tau}$ oscillations in the Super-Kamiokande atmospheric neutrino experiment," Physical Review D, vol. 60, Article ID 053006, 1999.

[14] M. Bahrami, S. Donadi, L. Ferialdi et al., "Are collapse models testable with quantum oscillating systems? The case of neutrinos, kaons, chiral molecules," Scientific Reports, vol. 3, article 1952, 2013.

[15] F.-C. Ma and H.-M. Hu, "Testing quantum mechanics in nuetrino oscillation," http://arxiv.org/abs/hep-ph/9805391.

[16] R. S. Raghavan, D. Minic, T. Takeuchi, and C. H. Tze, "Using neutrinos to test the time-energy uncertainty relation in an extreme regime," http://arxiv.org/abs/1210.5639.

[17] M. M. Guzzo, H. Nunokawa, and R. Tomàs, "Testing the principle of equivalence by supernova neutrinos," Astroparticle Physics, vol. 18, no. 3, pp. 277-286, 2002.

[18] L. Anchordoqui and F. Halzen, "IceHEP high energy physics at the South Pole," Annals of Physics, vol. 321, no. 11, pp. 2660-2716, 2006.
[19] M. Sprenger, P. Nicolini, and M. Bleicher, "Quantum gravity signals in neutrino oscillations," International Journal of Modern Physics E, vol. 20, no. S2, 2011.

[20] M. Sprenger, M. Bleicher, and P. Nicolini, "Neutrino oscillations as a novel probe for a minimal length," Classical and Quantum Gravity, vol. 28, Article ID 235019, 2011.

[21] C. Giunti and C. W. Kim, "Coherence of neutrino oscillations in the wave packet approach," Physical Review D, vol. 58, Article ID 017301, 1998.

[22] M. Beuthe, "Oscillations of neutrinos and mesons in quantum field theory," Physics Reports A, vol. 375, no. 2-3, pp. 105-218, 2003.

[23] J. Kersten, “Coherence of supernova neutrinos," Nuclear Physics B-Proceedings Supplements, vol. 237-238, pp. 342-344, 2013.

[24] Y. Farzan and A. Y. Smirnov, "Coherence and oscillations of cosmic neutrinos," Nuclear Physics B, vol. 805, no. 1-2, pp. 356376, 2008.

[25] J. Christian, "Testing gravity-driven collapse of the wave function via cosmogenic neutrinos," Physical Review Letters, vol. 95, Article ID 160403, 2005.

[26] S. Donadi, A. Bassi, L. Ferialdi, and C. Curceanu, "The effect of spontaneous collapses on neutrino oscillations," Foundations of Physics, vol. 43, no. 9, pp. 1066-1089, 2013.

[27] L. Diósi, "Gravitation and quantum-mechanical localization of macro-objects," Physics Letters A, vol. 105, no. 4-5, pp. 199-202, 1984.

[28] L. Diósi, "A universal master equation for the gravitational violation of quantum mechanics," Physics Letters A, vol. 120, pp. 377-381, 1987.

[29] L. Diósi, "Models for universal reduction of macroscopic quantum fluctuations," Physical Review A, vol. 40, no. 3, pp. 1165-1174, 1989.

[30] R. Penrose, "On gravity's role in quantum state reduction," General Relativity and Gravitation, vol. 28, no. 5, pp. 581-600, 1996.

[31] D. V. Ahluwalia and C. Burgard, "Interplay of gravitation and linear superposition of different mass eigenstates," Physical Review D, vol. 57, pp. 4724-4727, 1998.

[32] A. Bassi and G. C. Ghirardi, "Dynamical reduction models," Physics Reports, vol. 379, no. 5-6, pp. 257-426, 2003.

[33] C. Corianò, L. Delle Rose, E. Gabrielli, and L. Trentadue, "Mass corrections to flavor-changing fermion-graviton vertices in the standard model," Physical Review D, vol. 88, no. 8, Article ID 085008, 12 pages, 2013.

[34] S. P. Mikheev and A. Y. Smirnov, "Resonant amplification of $v$ oscillations in matter and solar-neutrino spectroscopy," Il Nuovo Cimento C, vol. 9, pp. 17-26, 1986.

[35] L. Wolfenstein, "Neutrino oscillations in matter," Physical Review D, vol. 17, no. 9, pp. 2369-2374, 1978.

[36] J. Beringer, J.-F. Arguin, R. M. Barnett et al., "Review of particle physics," Physical Review D, vol. 86, Article ID 010001, 2012.

[37] T. Ohlsson and H. Snellman, "Three flavor neutrino oscillations in matter," Journal of Mathematical Physics, vol. 41, no. 5, pp. 2768-2788, 2000, Erratum in Journal of Mathematical Physics, vol. 42, p. 2345, 2001.

[38] J. Edsjo, WimpSim Neutrino Monte Carlo, http://www.fysik.su .se/ edsjo/wimpsim.

[39] J. Gava, J. Kneller, C. Volpe, and G. C. McLaughlin, "Dynamical collective calculation of supernova neutrino signals," Physical Review Letters, vol. 103, Article ID 071101, 2009. 
[40] D. Vaananen and C. Volpe, "The neutrino signal at HALO: learning about the primary supernova neutrino fluxes and neutrino properties," Journal of Cosmology and Astroparticle Physics, vol. 2011, article 019, 2011.

[41] E. K. Akhmedov and A. Wilhelm, "Quantum field theoretic approach to neutrino oscillations in matter," Journal of High Energy Physics, vol. 2013, article 165, 2013.

[42] E. K. Akhmedov and J. Kopp, "Neutrino oscillations: quantum mechanics vs. quantum field theory," Journal of High Energy Physics, vol. 2010, no. 4, article 008, 2010, Erratum in: Journal of High Energy Physics, vol. 2013, no. 10, article 052, 2013.

[43] M. Blennow and A. Y. Smirnov, "Neutrino propagation in matter," Advances in High Energy Physics, vol. 2013, Article ID 972485, 33 pages, 2013.

[44] A. B. Balantekin and J. F. Beacom, "Semiclassical treatment of matter-enhanced neutrino oscillations for an arbitrary density profile," Physical Review D, vol. 54, pp. 6323-6337, 1996.

[45] A. S. Dighe, Q. Y. Liu, and A. Y. Smirnov, "Coherence and the day-night asymmetry in the solar neutrino flux," http://arxiv .org/abs/hep-ph/9903329.

[46] K. Kiers, S. Nussinov, and N. Weiss, "Coherence effects in neutrino oscillations," Physical Review D, vol. 53, pp. 537-547, 1996.

[47] D. G. Boulware and S. Deser, "Classical general relativity derived from quantum gravity," Annals of Physics, vol. 89, pp. 193-240, 1975.

[48] A. D. Dolgov and D. Ejlli, "Relic gravitational waves from light primordial black holes," Physical Review D, vol. 84, Article ID 024028, 2011.

[49] B. M. Barker, S. N. Gupta, and J. Kaskas, "Graviton bremsstrahlung and infrared divergence," Physical Review, vol. 182, no. 5, pp. 1391-1396, 1969.

[50] H. K. Tseng and R. H. Pratt, "Electron bremsstrahlung energy spectra above 2 MeV," Physical Review A, vol. 19, no. 4, pp. 1525$1528,1979$.

[51] D. H. Jakubassa-Amundsen, "High-energy bremsstrahlung from polarized electrons colliding with spin-1/2 nuclei," Physical Review C, vol. 87, Article ID 064609, 2013.

[52] D. N. Kabat and M. Ortiz, "Eikonal quantum gravity and planckian scattering," Nuclear Physics B, vol. 388, no. 2, pp. 570592, 1992.

[53] N. A. Voronov, "Gravitational Compton effect and photoproduction of gravitons by electrons," Zhurnal Éksperimental'nŏ $i$ Teoreticheskŏ̌ Fiziki, vol. 64, pp. 1889-1901, 1973.

[54] M. C. Gonzalez-Garcia, M. Maltoni, J. Salvado, and T. Schwetz, "Global fit to three neutrino mixing: critical look at present precision," Journal of High Energy Physics, vol. 2012, no. 12, article 123, 2012.

[55] A. Strumia and F. Vissani, "Neutrino masses and mixings and...," http://arxiv.org/abs/hep-ph/0606054.

[56] C. W. Kim and A. Pevsner, Neutrinos in Physics and Astrophysics, Harwood Academic, New York, NY, USA, 1993.

[57] K.-C. Lai, G.-L. Lin, and T. C. Liu, "Flavor transition mechanisms of propagating astrophysical neutrinos: a model independent parametrization," Physical Review D, vol. 82, Article ID 103003, 2010.

[58] S. Choubey and W. Rodejohann, "Flavor composition of ultrahigh energy neutrinos at source and at neutrino telescopes," Physical Review D, vol. 80, Article ID 113006, 2009.

[59] A. Esmaili and Y. Farzan, "An analysis of cosmic neutrinos: flavor composition at source and neutrino mixing parameters," Nuclear Physics B, vol. 821, pp. 197-214, 2009.
[60] K. Scholberg, "Supernova neutrino detection," Annual Review of Nuclear and Particle Science, vol. 62, pp. 81-103, 2012.

[61] T. Akiri, D. Allspach, M. Andrews et al., "The 2010 interim report of the long-baseline neutrino experiment collaboration physics working groups," http://arxiv.org/abs/1110.6249.

[62] P. A. N. Machado, T. Muhlbeier, H. Nunokawa, and R. Z. Funchal, "Potential of a neutrino detector in the ANDES underground laboratory for geophysics and astrophysics of neutrinos," Physical Review D, vol. 86, no. 12, Article ID 125001, 19 pages, 2012.

[63] J. Miller and R. Pasechnik, "Quantum gravity effect on neutrino oscillations in a strong gravitational field," http://arxiv.org/abs/ 1305.4430 .

[64] R. Paszko and A. Accioly, "Equivalence between the semiclassical and effective approaches to gravity," Classical and Quantum Gravity, vol. 27, no. 14, Article ID 145012, 2010.

[65] R. J. Gould, "The graviton luminosity of the sun and other stars," The Astrophysical Journal, vol. 288, pp. 789-794, 1985.

[66] B. M. Barker and S. N. Gupta, "High-energy graviton bremsstrahlung," Physical Review D, vol. 9, no. 2, pp. 334-338, 1974.

[67] P. C. Peters, "Relativistic gravitational bremsstrahlung," Physical Review D, vol. 1, no. 6, pp. 1559-1571, 1970.

[68] D. V. Ahluwalia, "On reconciling atmospheric, lsnd, and solar neutrino-oscillation data," Modern Physics Letters A, vol. 13, no. 28, pp. 2249-2264, 1998.

[69] H.-T. Janka, "Explosion mechanisms of core-collapse supernovae," Annual Review of Nuclear and Particle Science, vol. 62, pp. 407-451, 2012.

[70] M. Schlosshauer, "Decoherence, the measurement problem, and interpretations of quantum mechanics," Reviews of Modern Physics, vol. 76, p. 1267, 2005.

[71] L. Stodolsky, "When the wavepacket is unnecessary," Physical Review D, vol. 58, Article ID 036006, 1998.

[72] E. Akhmedov, J. Kopp, and M. Lindner, "Decoherence by wave packet separation and collective neutrino oscillations," http://arxiv.org/abs/1405.7275. 

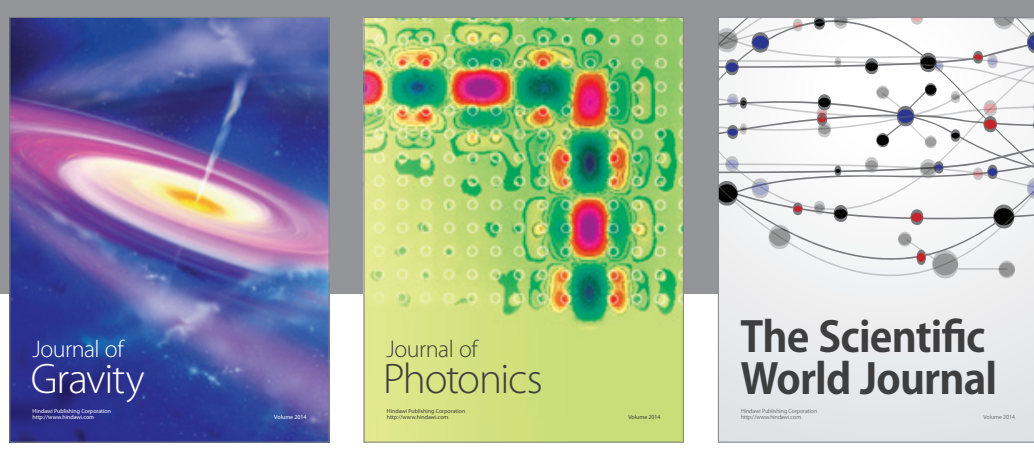

The Scientific World Journal
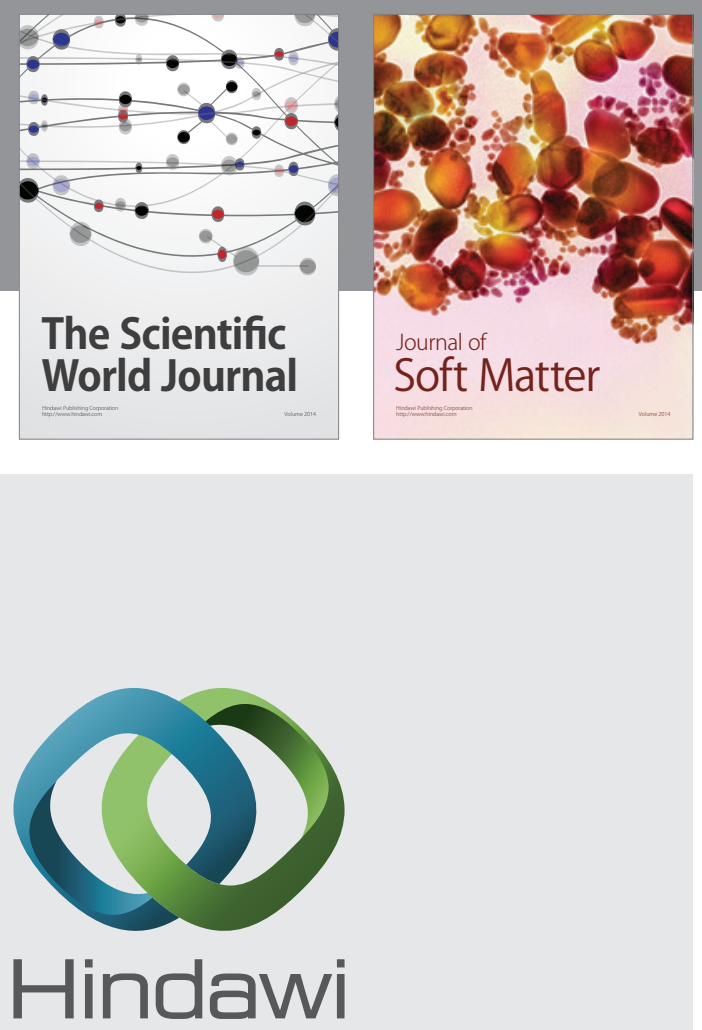

Submit your manuscripts at

http://www.hindawi.com

nternational Journal of

Statistical Mechanics
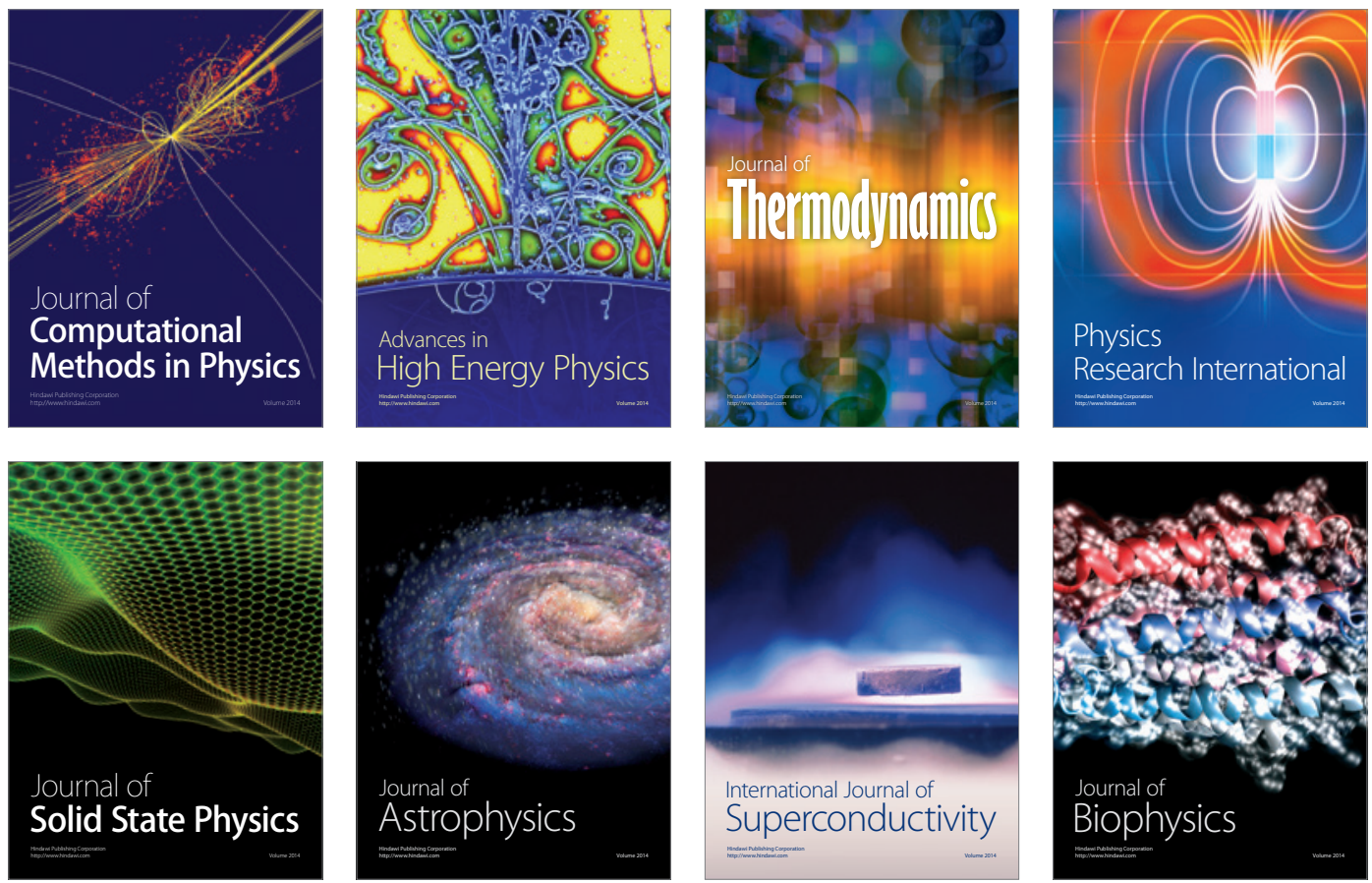
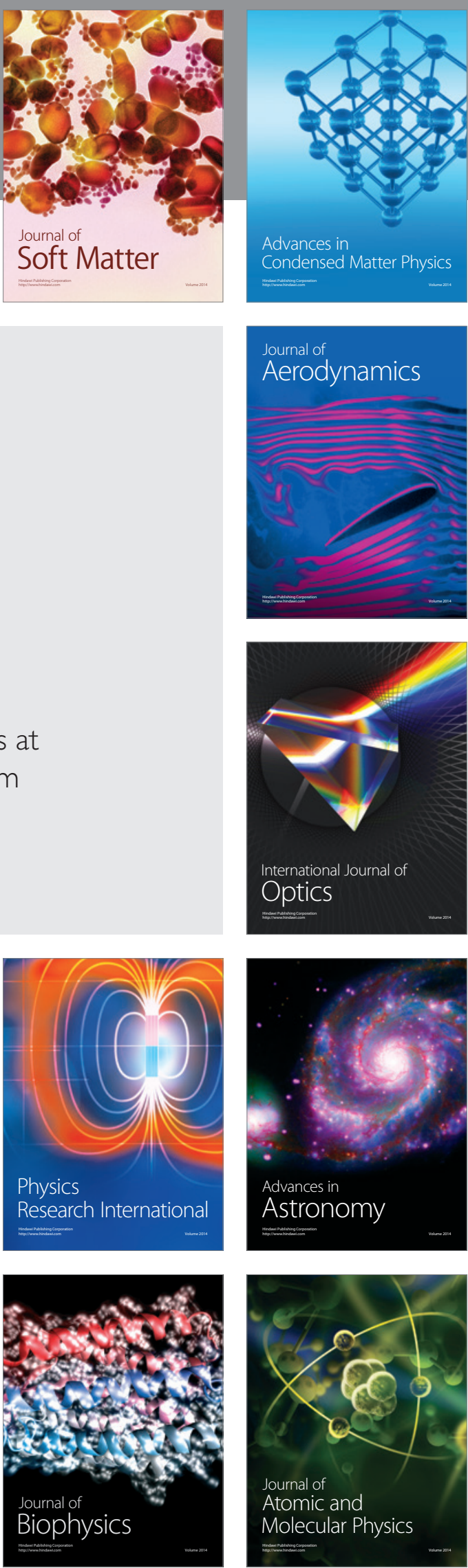